\title{
Gene content evolution in the arthropods
}

Gregg W. C. Thomas' ${ }^{1}$ Elias Dohmen 2,3,4, Daniel S. T. Hughes ${ }^{5,6}$, Shwetha C. Murali ${ }^{5,7}$, Monica Poelchau ${ }^{8}$, Karl Glastad ${ }^{9,10}$, Clare A. Anstead ${ }^{11}$, Nadia A. Ayoub ${ }^{12}$, Phillip Batterham ${ }^{13}$, Michelle Bellair ${ }^{5,14}$, Greta J. Binford ${ }^{15}$, Hsu Chao ${ }^{5}$, Yolanda H. Chen ${ }^{16}$, Christopher Childers ${ }^{8}$, Huyen Dinh ${ }^{5}$, Harsha Vardhan Doddapaneni ${ }^{5}$, Jian J. Duan ${ }^{17}$, Shannon Dugan ${ }^{5}$, Lauren A. Esposito ${ }^{18}$, Markus Friedrich ${ }^{19}$, Jessica Garb ${ }^{20}$, Robin B. Gasser ${ }^{11}$,

Michael A. D. Goodisman ${ }^{9}$ Dawn E. Gundersen-Rindal2 ${ }^{21}$, Yi Han ${ }^{5}$, Alfred M. Handler ${ }^{22}$, Masatsugu Hatakeyama ${ }^{23}$, Lars Hering ${ }^{24}$, Wayne B. Hunter ${ }^{25}$, Panagiotis loannidis ${ }^{26,27}$, Joy C. Jayaseelan ${ }^{5}$, Divya Kalra ${ }^{5}$, Abderrahman Khila ${ }^{28}$, Pasi K. Korhonen ${ }^{11}$, Carol Eunmi Lee ${ }^{29}$, Sandra L. Lee ${ }^{5}$, Yiyuan Li ${ }^{30}$, Amelia R. I. Lindsey ${ }^{31,32}$, Georg Mayer ${ }^{24}$, Alistair P. McGregor ${ }^{33}$, Duane D. McKenna ${ }^{34}$, Bernhard Misof ${ }^{35}$, Mala Munidasa ${ }^{5}$, Monica Munoz-Torres ${ }^{36,37}$, Donna M. Muzny ${ }^{5}$, Oliver Niehuis ${ }^{38}$, Nkechinyere Osuji-Lacy ${ }^{5}$, Subba R. Palli ${ }^{39}$, Kristen A. Panfilio ${ }^{40}$, Matthias Pechmann ${ }^{41}$, Trent Perry ${ }^{13}$, Ralph S. Peters ${ }^{42}$, Helen C. Poynton ${ }^{43}$, Nikola-Michael Prpic ${ }^{44,45}$, Jiaxin Qu ${ }^{5}$, Dorith Rotenberg ${ }^{46}$, Coby Schal ${ }^{47}$, Sean D. Schoville ${ }^{48}$, Erin D. Scully ${ }^{49}$, Evette Skinner ${ }^{5}$, Daniel B. Sloan ${ }^{50}$, Richard Stouthamer ${ }^{31}$, Michael R. Strand ${ }^{51}$, Nikolaus U. Szucsich ${ }^{52}$, Asela Wijeratne ${ }^{34,53}$, Neil D. Young ${ }^{11}$, Eduardo E. Zattara ${ }^{54}$, Joshua B. Benoit ${ }^{55}$, Evgeny M. Zdobnov ${ }^{26}$, Michael E. Pfrender ${ }^{30}$, Kevin J. Hackett ${ }^{56}$, John H. Werren ${ }^{57}$, Kim C. Worley ${ }^{5}$, Richard A. Gibbs ${ }^{5}$, Ariel D. Chipman ${ }^{58}$, Robert M. Waterhouse ${ }^{59}$, Erich Bornberg-Bauer ${ }^{2,3,60}$, Matthew W. Hahn ${ }^{1}$ and Stephen Richards ${ }^{5,61 *}$ (D)

\section{Abstract}

Background: Arthropods comprise the largest and most diverse phylum on Earth and play vital roles in nearly every ecosystem. Their diversity stems in part from variations on a conserved body plan, resulting from and recorded in adaptive changes in the genome. Dissection of the genomic record of sequence change enables broad questions regarding genome evolution to be addressed, even across hyper-diverse taxa within arthropods.

Results: Using 76 whole genome sequences representing 21 orders spanning more than 500 million years of arthropod evolution, we document changes in gene and protein domain content and provide temporal and phylogenetic context for interpreting these innovations. We identify many novel gene families that arose early in the evolution of arthropods and during the diversification of insects into modern orders. We reveal unexpected variation in patterns of DNA methylation across arthropods and examples of gene family and protein domain evolution coincident with the appearance of notable phenotypic and physiological adaptations such as flight, metamorphosis, sociality, and chemoperception.

Conclusions: These analyses demonstrate how large-scale comparative genomics can provide broad new insights into the genotype to phenotype map and generate testable hypotheses about the evolution of animal diversity.

Keywords: Arthropods, Genome assembly, Genomics, Protein domains, Gene content, Evolution, DNA methylation

\footnotetext{
* Correspondence: srichards@ucdavis.edu

${ }^{5}$ Human Genome Sequencing Center, Department of Human and Molecular Genetics, Baylor College of Medicine, One Baylor Plaza, Houston, TX 77030, USA

${ }^{61}$ Present Address: UC Davis Genome Center, University of California, Davis,

CA 95616, USA

Full list of author information is available at the end of the article
}

(c) The Author(s). 2020 Open Access This article is distributed under the terms of the Creative Commons Attribution 4.0 International License (http://creativecommons.org/licenses/by/4.0/), which permits unrestricted use, distribution, and reproduction in any medium, provided you give appropriate credit to the original author(s) and the source, provide a link to the Creative Commons license, and indicate if changes were made. The Creative Commons Public Domain Dedication waiver (http://creativecommons.org/publicdomain/zero/1.0/) applies to the data made available in this article, unless otherwise stated. 


\section{Background}

Arthropods (chelicerates, myriapods, crustaceans, and hexapods) constitute the most species-rich and diverse phylum on Earth, having adapted, innovated, and expanded into all major habitats within all major ecosystems. They are found as carnivores, detritivores, herbivores, and parasites. As major components of the world's biomass, their diversity and ubiquity lead naturally to significant interactions with humanity, as crop pests, disease vectors, food sources, pollinators, and synanthropes. Despite their diversity, arthropods share a deeply conserved and highly modular body plan. They are bilaterally symmetrical, with serially repeated segments along the anterior-posterior axis. Many segments bear paired appendages, which can take the form of antennae, feeding appendages, gills, and jointed legs. Many arthropods have evolved specialized secretions such as venom or silk, extruded from dedicated structures that further capitalize on this segmental modularity. Arthropods also have a hard exoskeleton, composed mostly of chitin, which molts as the animal grows in size. One group of arthropods, the winged insects (Pterygota), took to the skies, bearing up to two pairs of wings as outgrowths of that exoskeleton.

The extraordinary diversity of arthropods is manifested in a series of genomic changes and innovations selected for throughout their evolutionary history. However, linking this phenotypic diversity to underlying genomic changes remains an elusive challenge. The major transitions in arthropod evolution include the differential grouping of body segments into morphological units with a common function (e.g., head, thorax, and abdomen in the Hexapoda) in different taxa, the independent and parallel colonizations of terrestrial and freshwater habitats by ancestrally marine lineages [1, 2], the emergence of active flight in insects [3, 4], and the evolution of insect metamorphosis [5]. Multiple genomic mechanisms might be responsible for such innovations, but the underlying molecular transitions have not been explored on a broad phylogenomic scale. Tracing these transitions at the genomic level requires mapping whole genome data to a robust phylogenetic framework. Here, we explore the evolution of arthropod genomes using a phylogeny-mapped genomic resource of 76 species representing the breath of arthropod diversity.

\section{Results}

\section{An arthropod evolution resource}

As a pilot project for the $15 \mathrm{~K}$ initiative to sequence 5000 arthropod genomes [6], we sequenced and annotated the genomes of 28 arthropod species (Additional file 1: Table S1). These include a combination of species of agricultural or ecological importance, emerging laboratory models, and species occupying key positions in the arthropod phylogeny. We combined these newly sequenced genomes with those of 48 previously sequenced arthropods creating a dataset comprising 76 species representing the four extant arthropod subphyla and spanning 21 taxonomic orders. Using the OrthoDB gene orthology database [7], we annotated 38,195 protein ortholog groups (orthogroups/gene families) among all 76 species (Fig. 1). Based on single-copy orthogroups within and between orders, we then built a phylogeny of all major arthropod lineages (Fig. 2). This phylogeny is mostly consistent with previous arthropod phylogenies [8-10], with the exception being that we recover a monophyletic Crustacea, rather than the generally accepted paraphyletic nature of Crustacea with respect to Hexapoda; the difference is likely due to our restricted taxon sampling (see "Methods"). We reconstructed the gene content and protein domain arrangements for all 38,195 orthogroups in each of the lineages for the 76 species in the arthropod phylogeny. This resource (available at https://arthrofam.org and Additional file 1: Table S11) forms the basis for the analyses detailed below and is an unprecedented tool for identifying and tracking genomic changes over arthropod evolutionary history.

\section{Genomic change throughout arthropod history}

Evolutionary innovation can result from diverse genomic changes. New genes can arise either by duplication or, less frequently, by de novo gene evolution [11]. Genes can also be lost over time, constituting an underappreciated mechanism of evolution [12, 13]. Protein domains are the basis of reusable modules for protein innovation, and the rearrangement of domains to form new combinations plays an important role in molecular innovation [14]. Together, gene family expansions and contractions and protein domain rearrangements may coincide with phenotypic innovations in arthropods. We therefore searched for signatures of such events corresponding with pivotal phenotypic shifts in the arthropod phylogeny.

Using ancestral reconstructions of gene counts (see "Methods"), we tracked gene family expansions and losses across the arthropod phylogeny. Overall, we inferred 181,157 gene family expansions and 87,505 gene family contractions. A total of 68,430 gene families were inferred to have gone extinct in at least one lineage, and 9115 families emerged in different groups. We find that, of the 268,662 total gene family changes, 5843 changes are statistically rapid (see "Methods"), with the German cockroach, Blattella germanica, having the most rapid gene family changes (Fig. 3e). The most dynamically changing gene families encode proteins involved in functions of xenobiotic defense (cytochrome P450s, sulfotransferases), digestion (peptidases), chitin exoskeleton structure and metabolism, multiple zinc finger 


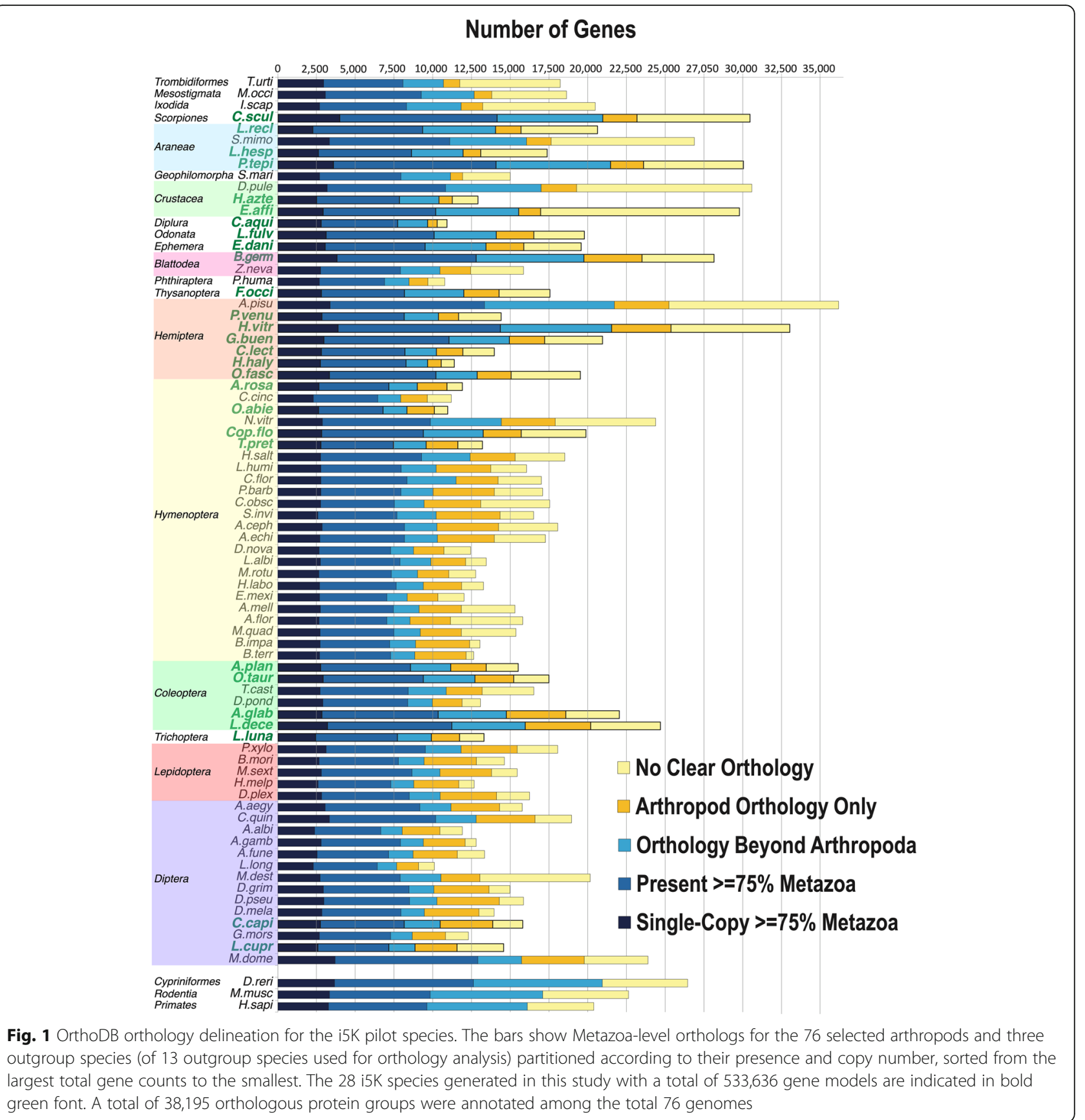

transcription factor types, HSP20 domain stress response, fatty acid metabolism, chemosensation, and ecdysteroid (molting hormone) metabolism (Additional file 1: Table S15). Using the estimates of where in the phylogeny these events occurred, we can infer characteristics of ancestral arthropods. For example, we identified 9601 genes in the last insect common ancestor (LICA) and estimate $\sim 14$, 700 LICA genes after correcting for unobserved gene extinctions (Fig. 2, Additional file 2: Figure S1 and Additional file 1: Table S16). We reconstructed similar numbers for ancestors of the six well-represented arthropod taxa in our sample (Fig. 2 and Additional file 1: Table S16). Of the 9601 genes present in LICA, we identified 147 emergent gene families (i.e., lineage-restricted families with no traceable orthologs in other clades) which appeared concurrently with the evolution of insects (Fig. 3a, Fig. 2 node 62, Additional file 1: Table S18). Gene Ontology term analysis of these 147 gene families recovered multiple key functions, including cuticle and cuticle development (suggesting changes in exoskeleton development), visual learning and behavior, pheromone and odorant binding (suggesting the ability to sense in 


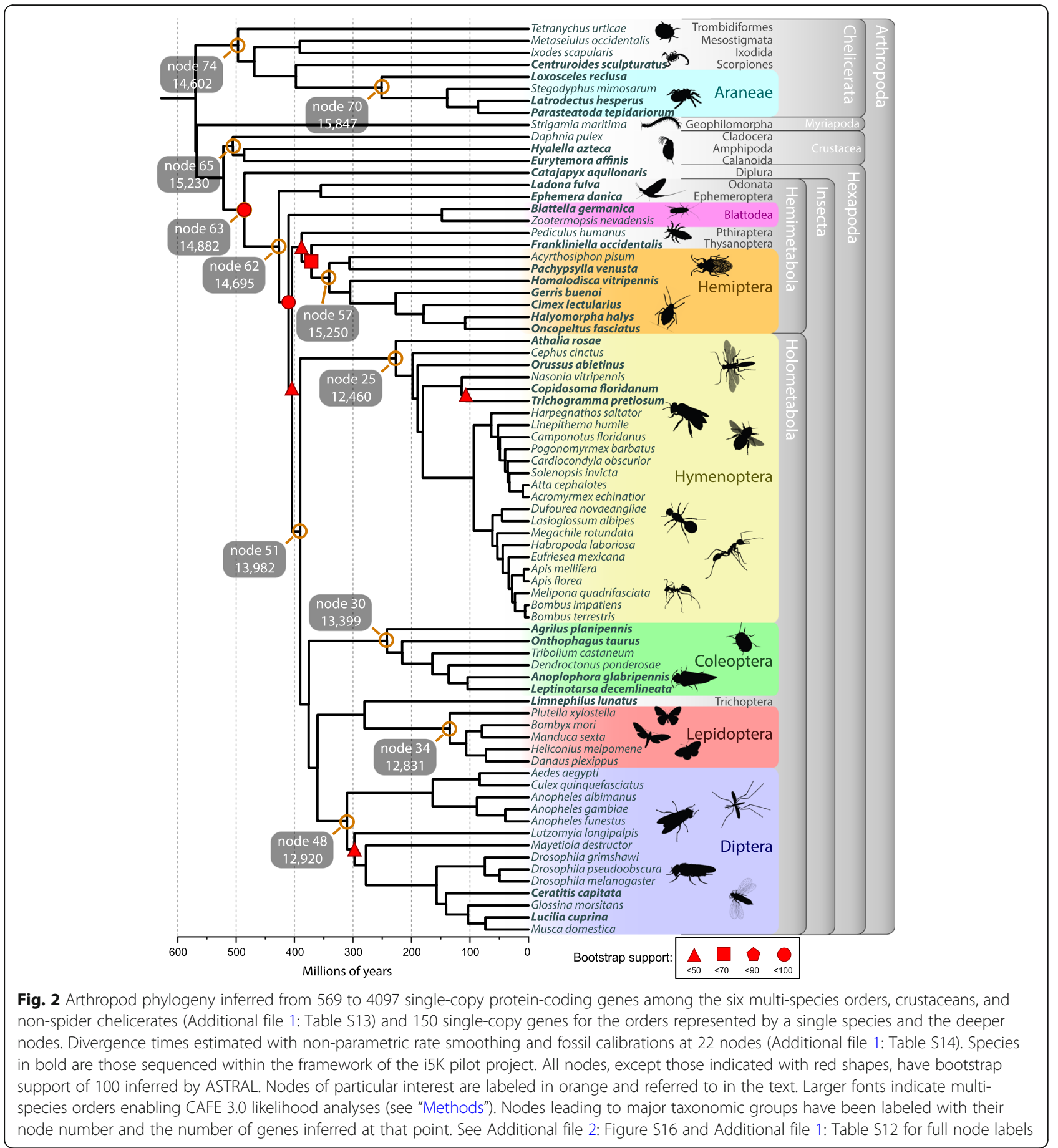

terrestrial/aerial environments rather than aquatic), ion transport, neuronal activity, larval behavior, imaginal disc development, and wing morphogenesis. These emergent gene families likely allowed insects to undergo substantial diversification by expanding chemical sensing, such as an expansion in odorant binding to locate novel food sources and fine-tune species self-recognition [15-17]. Others, such as cuticle proteins underlying differences in exoskeleton structure, may enable cuticle properties optimized for diverse environmental habitats or life history stages [18]. In contrast, the data reveal only ten gene families that arose along the ancestral lineage of the Holometabola (Fig. 3b, Additional file 1: Table S19), implying that genes and processes required for the transition to holometabolous development, such as imaginal disc development, were already present in the hemimetabolous 


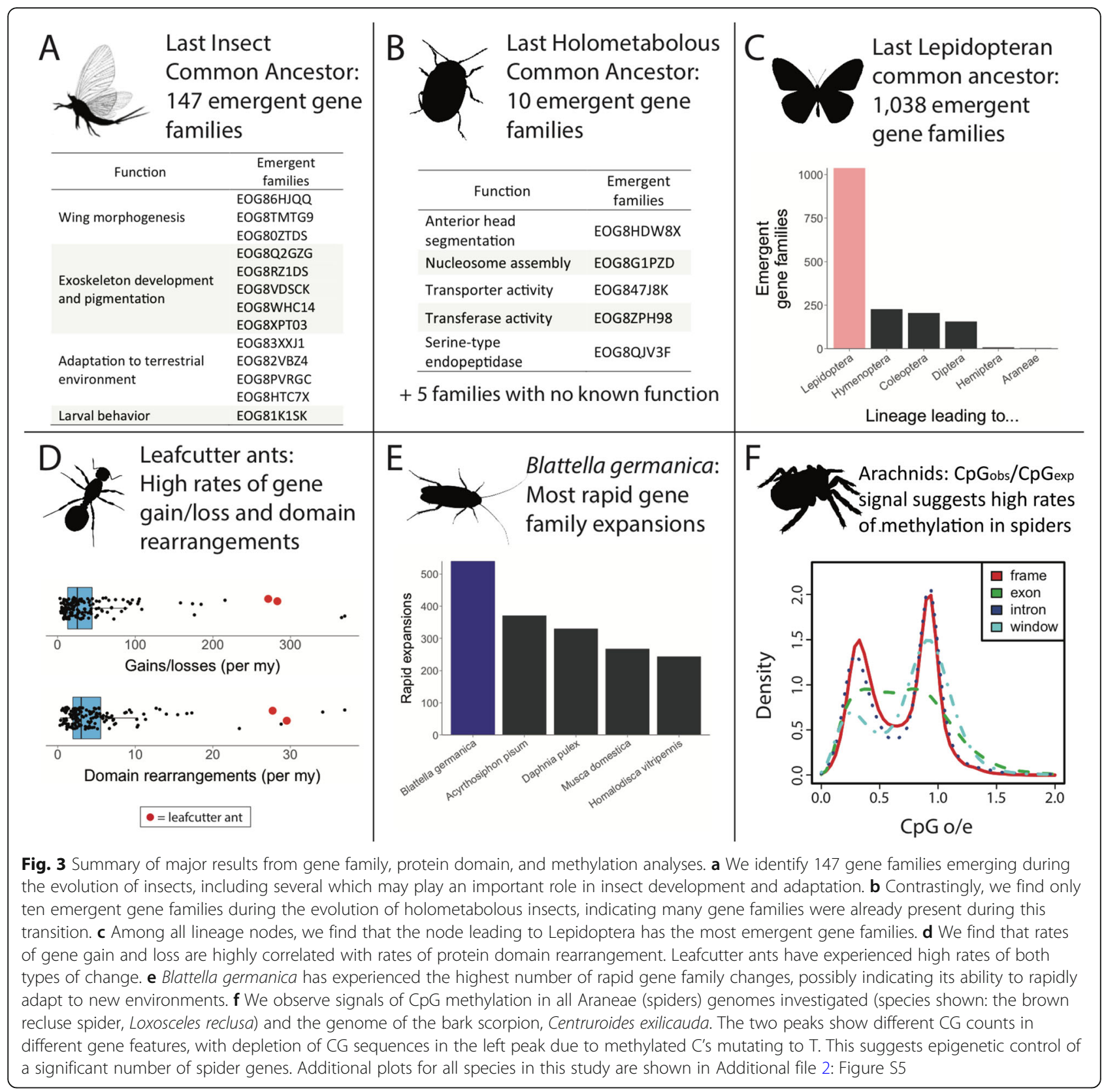

ancestors. This is consistent with Truman and Riddiford's model that the holometabolous insect larva corresponds to a late embryonic state of hemimetabolous insects [19].

We identified numerous genes that emerged in specific orders of insects. Strikingly, we found 1038 emergent gene families in the first ancestral Lepidoptera node (Fig. 3c). This node has by far the most emergent gene families, with the next highest being the node leading to the bumble bee genus Bombus with 860 emergent gene families (Additional file 2: Figure S2). Emergent lepidopteran gene families show enrichment for functional categories such as peptidases and odorant binding. Among the other insect orders, we find 227 emergent families in the node leading to the Hymenoptera, 205 in that leading to Coleoptera, and 156 in that leading to Diptera. Though our sampling is extensive, it is possible that gene families we have classified as emergent may be present in unsampled lineages.

Similarly, we reconstructed the protein domain arrangements for all nodes of the arthropod phylogeny, that is, the permutations in protein domain type per (multi-domain) gene. In total, we can explain the underlying events for more than 40,000 domain arrangement changes within the arthropods. The majority of domain arrangements ( $48 \%$ of all observable events) were formed by a fusion of two ancestral arrangements, while the 
fission of an existing arrangement into two new arrangements accounts for $14 \%$ of all changes. Interestingly, $37 \%$ of observed changes can be explained by losses (either as part of an arrangement (14\%) or the complete loss of a domain in a proteome $(23 \%)$ ), while emergence of a novel protein domain is a very rare event, comprising only $1 \%$ of total events.

We observe high concordance between rates of gene family dynamics and protein domain rearrangement (Fig. 4 and Additional file 2: Figure S3). In some cases, we find specific examples of overlap between gene family and protein domain evolution. For example, spiders have the characteristic ability to spin silk and are venomous. Correspondingly, we identify ten gene families associated with venom or silk production that are rapidly expanding within Araneae (spiders, Additional file 1: Table S20). In parallel, we find a high rate of new protein domains in the subphylum Chelicerata, including a large number within Araneae associated with venom and silk production. For example, "spider silk protein 1" (Pfam ID: PF16763), "Major ampullate spidroin 1 and 2" (PF11260), "Tubuliform egg casing silk strands structural domain" (PF12042), and "Toxin with inhibitor cystine knot ICK or Knottin scaffold" (PF10530) are all domains that emerged within the spider clade. Venom domains also emerged in other venomous chelicerates, such as the bark scorpion, Centruroides sculpturatus.

We identified gene family changes that may underlie unique phenotypic transitions. The evolution of eusociality among three groups in our study, bees and ants (both Hymenoptera), and termites (Blattodea), requires these insects to be able to recognize other individuals of their colony (such as nest mates of the same or different caste), or invading individuals (predators, slave-makers and hosts) for effective coordination. We find 41 functional terms enriched for gene family changes in all three groups, with multiple gene family gains related to olfactory reception and odorant binding (Additional file 1: Table S21) in agreement with previous chemoreceptor studies of these species [20,21].

Finally, we observe species-specific gene family expansions that suggest biological functions under selection. The German cockroach, a pervasive tenant in human dwellings across the world, has experienced the highest

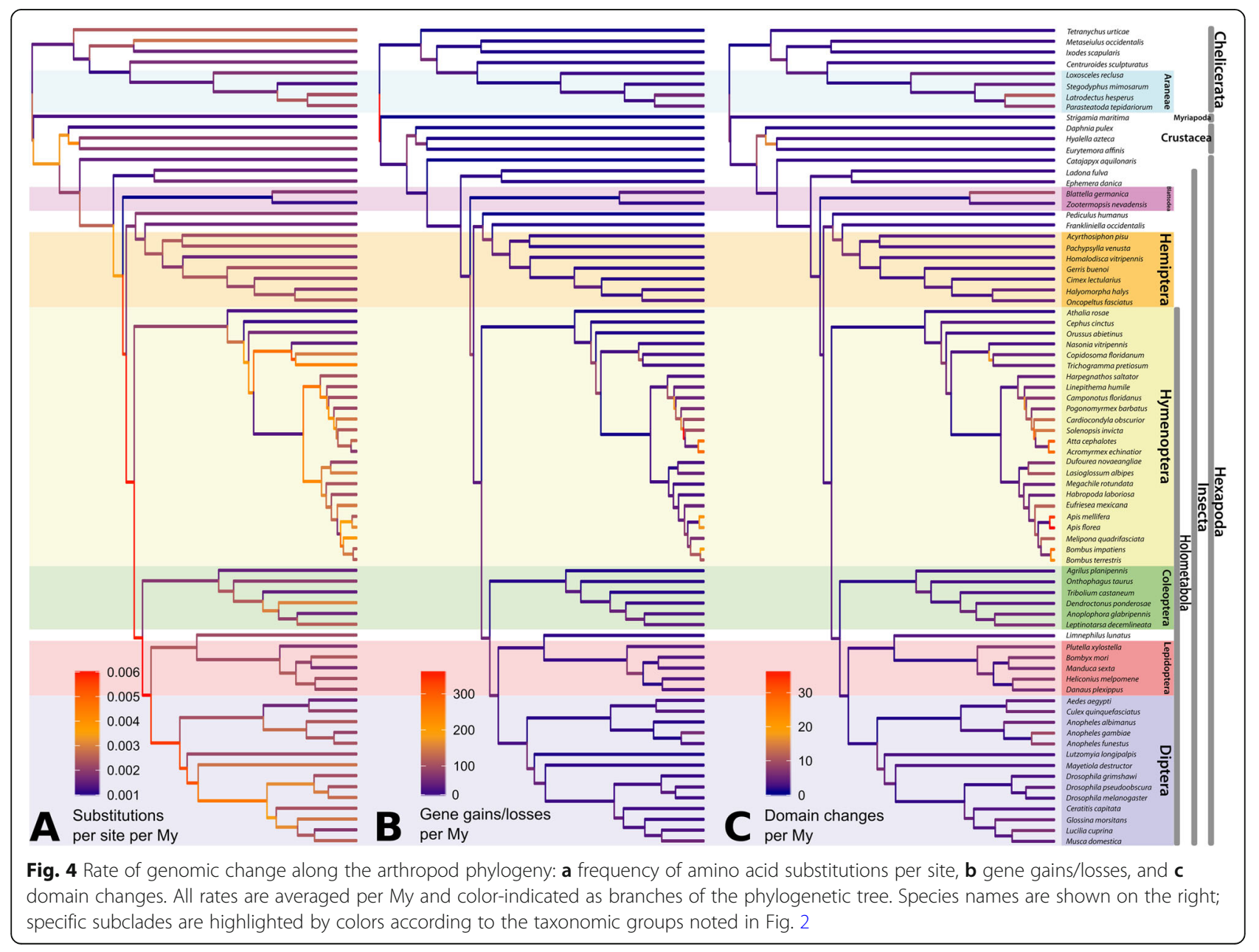


number of rapidly evolving gene families among the arthropods studied here, in agreement with a previously reported major expansion of chemosensory genes [22]. We also find the largest number of domain rearrangement events in B. germanica. The impressive capability of this cockroach to survive many environments and its social behavior could be linked to these numerous and rapid evolutionary changes at the genomic level and warrants more detailed investigation.

\section{Evolutionary rates within arthropod history}

The rate of genomic change can reflect key events during evolution along a phylogenic lineage. Faster rates might imply small population sizes or strong selective pressure, possibly indicative of rapid adaptive radiations, and slower rates may indicate stasis. Studying rates of change requires a time-calibrated phylogeny. For this, we used 22 fossil calibration points $[8,23]$ and obtained branch lengths for our phylogeny in millions of years (My) (Fig. 2) that are very similar to those obtained by Misof et al. [8] and Rota-Stabelli et al. [9].

We examined the rates of three types of genomic change: (i) amino acid substitutions, (ii) gene duplications and gene losses, and (iii) protein domain rearrangements, emergence, and loss. While clearly not changing in a clock-like manner, all types of genomic change have a strikingly small amount of variation in rate among the investigated species (Fig. 4). We estimate an average amino acid substitution rate of $2.54 \times 10^{-3}$ substitutions per site per My with a standard deviation of $1.11 \times 10^{-3}$. The slowest rate is found in the branch leading to the insect order Blattodea (cockroaches and termites), while the fastest rates are found along the short branches during the early diversification of Holometabola, suggesting a period of rapid evolution, a pattern similar to that found for amino acid sequence evolution during the Cambrian explosion [24]. Other branches with elevated amino acid divergence rates include those leading to Acarina (mites), and to the Diptera (flies).

Though we observe thousands of genomic changes across the arthropod phylogeny, they are mostly evenly distributed (Fig. 3d). Rates of gene duplication and loss show remarkably little variation, both across the tree and within the six multi-species orders (Additional file 1: Table S13). Overall, we estimate an average rate of 43.0 gains/losses per My, but with a high standard deviation of 59.0 that is driven by a few lineages with greatly accelerated rates. Specifically, the terminal branches leading to the leafcutter ants Atta cephalotes and Acromyrmex echinatior along with the internal node leading to the leafcutter ants and the red fire ant (node HY29) have exceptionally high gene gain/loss rates of 266,277 , and 370 per My, respectively (Fig. 3d). This is an order of magnitude higher than average, as previously reported among leafcutter ants [25]. Removing these nodes, the average becomes 27.2 gains/losses per My (SD 19.7). Interestingly, the high gain/loss rates observed in these ants, in contrast to other arthropods, are not due to large gene content change in a small number of gene families. They are instead due mostly to single gene gains or losses in a large number of gene families.

Regarding protein domain rearrangements, which mainly arise from duplication, fusion and terminal losses of domains [26], we estimate an average rate of 5.27 events per My, approximately eightfold lower than the rate of gene gain/loss. Interestingly, we discovered a strong correlation between rates of gene gain/loss and domain rearrangement (Figs. 3d and 4 and Additional file 2: Figure S3). For example, terminal branches within the Hymenoptera have an accelerated rate of domain rearrangement, which coincides with the increased rate of gene gains and losses observed along those branches. This novel finding is surprising, given that these processes follow largely from different underlying genetic events (see [27] for discussion of these processes).

Our examination found no correlation between variation in amino acid substitution rates and rates of gene gain/loss or domain rearrangement rates (Fig. 4 and Additional file 2: Figure S3). Branches with accelerated rates of amino acid substitution, such as the lineage leading to the most recent common ancestor of the insect superorder Holometabola, do not show corresponding increases in gene gain/loss rates. Similarly, the hymenopteran lineages displaying the fastest rate of gene gain/loss in our analysis do not display higher rates of amino acid substitutions.

\section{Control of novel genes: methylation signals in arthropod genomes}

Our description of gene family expansions in arthropods by gene duplication naturally suggests the need for differential control of duplicated genes. Insect epigenetic control by $\mathrm{CpG}$ methylation is important for caste development in honey bees [28] and polyphenism in aphids [29]. However, signals of methylation are not seen in every insect, and the entire Dipteran order appears to have lost the capacity for DNA methylation. Given this diversity in the use of, and capacity for epigenetic control by DNA methylation, we searched for signals of CpG methylation in our broader sampling of arthropod genomes. We find several independent losses of the DNA methylation machinery across the arthropods (Additional file 2: Figure S4) [30]. This indicates that DNA methylation is not universally necessary for development and that the DNA methyltransfereases in insects may function in ways not previously appreciated [31]. Additionally, putative levels of DNA methylation vary 
considerably across arthropod species (Additional file 2: Figures S4, S5). Notably, the hemimetabolous insects and non-insect arthropods show higher levels of DNA methylation signals than the holometabolous insects [30]. Araneae (spiders), in particular, show clear bimodal patterns of methylation (Fig. 3f and Additional file 2: Figure S5), with some genes displaying high methylation signals and others not. A possible connection between spider bimodal gene methylation and their proposed ancestral whole genome duplication will require additional investigation. This pattern is also found in some holometabolous insects, suggesting that the division of genes into methylated and unmethylated categories is a relatively ancient trait in Arthropoda, although many species have since lost this clear distinction. Finally, some taxa, particularly in Hymenoptera, show higher levels of CpG di-nucleotides than expected by chance alone, which may be a signal of strong effects of gene conversion in the genome [32].

\section{Discussion}

The i5K pilot initiative has assembled an unparalleled genomic dataset for arthropod research and conducted a detailed phylogenetic analysis of evolutionary changes at the genomic level within this diverse and fascinating phylum. The combined research output of species-level i5K work has been substantial and wide-ranging, addressing pests of agricultural crops [33, 34] and animals [35], urban [20,36] and forest [37] pests, biocontrol species [38], along with developmental models [18, 39, 40], indicators of water quality and models for toxicology $[15,41]$ (Additional file 1: Table S1).

Here, in contrast, we take a broad overview generating a comparative genomics resource for a phylum with an evolutionary history of over 500 million years. Our analyses identify multiple broad patterns such as the very small number of novel protein domains and a surprising lack of variation in the rates of some types of genomic change. We pinpoint the origin of specific gene families and trace key transitions during which specific gene families or protein domains have undergone rapid expansions or contractions. An overview of the diversity and evolution of TEs found large intra- and inter-lineage variation in both TE content and composition [42].

Nonetheless, drawing functional biological conclusions from these data is not straightforward. In some cases, the link between specific gene families and their biological function is clear. This is true for genes related to specific physiological functions (e.g., olfaction) or to the production of specific compounds (e.g., silk or venom). However, for many gene families, there is no known function, highlighting the need for functional genomic studies. For example, emergent gene families such as those identified in the Lepidoptera, and rapidly evolving and diverging gene families, cannot be studied in the dipteran Drosophila model.

A key consequence of the relatively stable rate of gene family and protein domain change across the arthropod tree is that major morphological transitions (e.g., full metamorphosis, wing emergence, Additional file 1: Table S17) could not easily be identified by surges in gene content or protein domain change. There are two possible exceptions in our data. We see an increased rate of gene family extinction along the ancestral nodes from the ancestor of the cockroach and termites and hemimetabolous insects to the ancestor of Lepidoptera and Diptera (Additional file 2: Figure S6), suggesting the possibility of evolution by gene loss [12, 43]. This rate increase is not seen in wing evolution. The second possible exception is that of whole genome duplications (as proposed in spiders [40]), when there is a temporary opening of the "evolutionary search space" of gene and protein domain content. This overall finding is in line with the emerging understanding that morphology is effected by complex gene networks, which are active mostly during ontogenetic processes [44], rather than by individual "morphology genes". Morphological innovations are often based on modulating the timing and location of expression, rewiring of existing gene networks, and assembling new networks using existing developmental toolkit genes [45]. The current study was unable to address the evolution of non-coding sequences such as enhancers, promoters, and small and other non-coding RNAs underlying these networks due to the lack of sequence conservation over large evolutionary distances; however, our results underscore their evolutionary importance.

The advent of affordable and widely transferable genomics opens up many avenues for evolutionary analyses. The genome is both the substrate and record of evolutionary change, and it encodes these changes, but the connection is far from simple. A better understanding of the genotype-phenotype map requires in-depth experimental studies to test hypotheses generated by genomic analyses, such as those presented here. The diversity of arthropods provides unparalleled taxonomic resolution for phenotypic change, which, combined with the experimental tractability of many arthropods, suggests a productive area of future research using and building upon the resource established herein.

\section{Conclusions}

We have generated annotated draft genome assemblies for 28 species sampled from across the phylum Arthropoda. Combined with previously sequenced genomes, we documented changes in gene and protein domain content across 76 species sampled from 21 orders, spanning more than 500 million years. The resulting Arthropod 
resource comprises reconstructed gene content and protein domain arrangements for 38,195 orthogroups at each node of the Arthropod phylogeny. It enables inference and identification of gene content in terms of both families and domains at ancestral phylogenetic nodes. Rates of gene content change and protein domain change appear to be correlated, but neither gene content change nor protein domain change rates are correlated with amino-acid change. This work is a first look at the history of arthropod gene evolution, and an example of the power of comparative genomic analysis in a phylogenetic context to illuminate the evolution of life on earth.

\section{Methods}

\section{Sequencing, assembly, and annotation}

Twenty-eight arthropod species were sequenced using Illumina short read technology. In total, 126 short read libraries were generated and sequenced to generate 4.9 $\mathrm{Tb}$ of raw nucleotide sequence (Additional file 1: Table $\mathrm{S} 2$ ). For individual species, reads were assembled using AllpathsLG [46, 47] followed by refinements employing Atlas-Link [48] and Gapfill [49]. Version 1.0 assemblies had minimum, mean, and maximum scaffold N50 lengths of $13.8 \mathrm{~kb}, 1.0 \mathrm{Mb}$, and 7.1 Mb (Additional file 1: Table S3). Following re-assembly and collapsing of unassembled haplotypes using Redundans [50], version 2.0. assemblies had minimum, mean, and maximum contig N50 lengths of $11.1 \mathrm{~kb}, 166.2 \mathrm{~kb}$, and $857.0 \mathrm{~kb}$ with a mean scaffold N50 lengths of $619 \mathrm{~kb}$ (Additional file 1: Table S3). The redundans software and new assemblies became available late in the project timeline, and thus automated gene annotations, orthologous gene family identification in OrthoDB, and analysis were performed on the Version 1 ALLPATHS-LG-based assemblies.

To support the annotation, RNAseq data were generated from 25 species for which no data were available (Additional file 1: Table S4). A MAKER [51] based automated annotation pipeline was applied to the 1.0 assembly of each species with species-specific input RNAseq data and alignment data from a non-redundant metazoan protein sequence set containing all available arthropod protein sequences (see Additional file 2: Supplementary methods). This pipeline was applied to 28 species with annotatable genome assemblies generating 533,636 gene models, with minimum, mean, and maximum gene model numbers of 10,901, 19,058, and 33,019 per species (Additional file 1: Table S5, see Additional file 1: Table S7 for completeness statistics). Many of these gene models were manually curated using the i5k Workspace@NAL [52]. Given the magnitude of this manual task, the greatest fraction of gene models manually confirmed for a species was $15 \%$. The analyses presented here were performed on the automatically generated gene models.

\section{Orthology prediction}

Orthology delineation is a cornerstone of comparative genomics, offering qualified hypotheses on gene function by identifying "equivalent" genes in different species. We used the OrthoDB [7] (www.orthodb.org) orthology delineation process that is based on the clustering of best reciprocal hits (BRHs) of genes between all pairs of species. Clustering proceeds first by triangulating all BRHs and then subsequently adding in-paralogous groups and singletons to build clusters of orthologous genes. Each of these ortholog groups represent all descendants of a single gene present in the genome of the last common ancestor of all the species considered for clustering [53].

The orthology datasets computed for the analyses of the 28 i5K pilot species, together with existing sequenced and annotated arthropod genomes were compiled from OrthoDB v8 [54], which comprises 87 arthropods and an additional 86 other metazoans (including 61 vertebrates). Although the majority of these gene sets were built using MAKER (Additional file 1: Table S6), variation in annotation pipelines and supporting data, introduce a potential source of technical gene content error in our analysis.

Orthology clustering at OrthoDB included ten of the i5K pilot species (Anoplophora glabripennis, Athalia rosae, Ceratitis capitata, Cimex lectularius, Ephemera danica, Frankliniella occidentalis, Ladona fulva, Leptinotarsa decemlineata, Orussus abietinus, Trichogramma pretiosum). The remaining $18 \mathrm{i} 5 \mathrm{~K}$ pilot species were subsequently mapped to OrthoDB v8 ortholog groups at several major nodes of the metazoan phylogeny. Orthology mapping proceeds by the same steps as for $\mathrm{BRH}$ clustering, but existing ortholog groups are only permitted to accept new members, i.e., the genes from species being mapped are allowed to join existing groups if the $\mathrm{BRH}$ criteria are met. The resulting ortholog groups of clustered and mapped genes were filtered to select all groups with orthologs from at least two species from the full set of 76 arthropods, as well as retaining all orthologs from any of 13 selected outgroup species for a total of 47,281 metazoan groups with orthologs from 89 species. Mapping was also performed for the relevant species at the following nodes of the phylogeny: Arthropoda (38,195 groups, 76 species); Insecta (37,079 groups, 63 species); Endopterygota (34,614 groups, 48 species); Arachnida (8806 groups, 8 species); Hemiptera (8692 groups, 7 species); Hymenoptera (21,148 groups, 24 species); Coleoptera (12,365 groups, 6 species); and Diptera (17,701, 14 species). All identified BRHs, amino acid sequence alignment results, and orthologous group 
classifications were made available for downstream analyses: http://ezmeta.unige.ch/i5k.

\section{Arthropod phylogeny}

We reconstructed the arthropod phylogeny (Fig. 2) using protein sequences from the 76 genomes. Six different phylogenetic reconstruction approaches generated a consistent relationship among the orders (see Supplemental Methods), corresponding with previously inferred arthropod phylogenies [8-10].

Of the six orders in our dataset represented by multiple species (Additional file 2: Figures S7-S12), relationships within the Araneae, Hemiptera, Coleoptera, and Lepidoptera were identical, regardless of the tree building method used. Within the Hymenoptera, the only disagreement between methods concerned the position of the parasitoid wasps within the Chalcidoidea, with three methods placing Copidosoma floridanum as sister to Nasonia vitripennis (in agreement with recent phylogenomic research [55]), and the three other methods placing C. floridanum as sister to Trichogramma pretiosum (Additional file 2: Figure S9). Within the Diptera, we obtained a sister group relationship between the sand fly, Lutzomyia longipalpis, and the Culicidae, but this was not a stable topology across methods (Additional file 2: Figure S12).

The most contentious nodes in the phylogeny involve the relationship of crustaceans and hexapods. We recover a monophyletic Crustacea that represent the sister clade to Hexapoda (Fig. 2), in contrast to recent analyses suggesting this group is paraphyletic in respect to Hexapoda [56]. However, an extensive phylogenetic investigation (Additional file 2: Supplementary Results, Additional file 2: Figure S13) shows that regardless of the inference method used, the relationships among the crustacean and hexapod lineages remain uncertain. Aside from these few discrepancies, branch support values across the tree were high for all tree building methods used. Even when bootstrap support was < $100 \%$, all methods still inferred the same topology among the species included. The most likely reason for the difference from the current consensus is poor taxon sampling. Importantly, remipedes (the possible sister group of the hexapods) are missing from our taxon sampling, as are mystacocarids, ostracods, and pentatomids, and may change this result to the current consensus when added as was seen in [56].

\section{Divergence time estimation}

Phylogenetic branch lengths calibrated in terms of absolute time are required to study rates of evolution and to reconstruct ancestral gene counts. We used a nonparametric method of tree smoothing implemented in the software r8s [57] to estimate these divergence times.
Fossil calibrations are required to scale the smoothed tree by absolute time. We relied on Wolfe et al.'s [23] aggregation of deep arthropod fossils with additional recent fossils used by Misof et al. [8] (Additional file 1: Table S14). The results indicate that the first split within arthropods (the chelicerate-mandibulate split) occurred $\sim 570$ million years ago (mya). We estimate that within the chelicerates, arachnids radiated from a common ancestor $\sim 500$ mya. Within the mandibulates, myriapods split from other mandibulates $\sim 570$ mya. Crustaceans started radiating $\sim 506$ mya, and insects started radiating $\sim 430$ mya.

\section{Substitution rate estimation}

To estimate substitution rates per year on each lineage of the arthropod phylogeny, we divided the expected number of substitutions (the branch lengths in the unsmoothed tree) by the estimated divergence times (the branch lengths in the smoothed tree) (Fig. 4).

\section{Gene family analysis}

With the 38,195 orthogroups and the ultrametric phylogeny, we were able to perform the largest gene family analysis of any group of taxa to date. In this analysis, we were able to estimate gene turnover rates $(\lambda)$ for the six multi-species taxonomic orders, to infer ancestral gene counts for each taxonomic family on each node of the tree, and to estimate gene gain/loss rates for each lineage of the arthropod phylogeny. The size of the dataset and the depth of the tree required several methods to be utilized.

Gene turnover rates $(\lambda)$ for the six multi-species orders were estimated with CAFE 3.0, a likelihood method for gene-family analysis [58]. CAFE 3.0 is able to estimate the amount of assembly and annotation error $(\varepsilon)$ present in the input gene count data. This is done by treating the observed gene family counts as distributions rather than certain observations. CAFE can then be run repeatedly on the input data while varying these error distributions to calculate a pseudo-likelihood score for each one. The error model that is obtained as the minimum score after such a search is then used by CAFE to obtain a more accurate estimate of $\lambda$ and reconstruct ancestral gene counts throughout the tree (Additional file 1: Table S12). However, with such deep divergence times of some orders, estimates of $\varepsilon$ may not be accurate. CAFE has a built-in method to assess significance of changes along a lineage given an estimated $\lambda$ and this was used to identify rapidly evolving families within each order. We partitioned the full dataset of 38,195 orthogroups for each order such that taxa not in the order were excluded for each family and only families that had genes in a given order were included in the analysis. This led to the counts of gene families seen in Additional file 1: Table S11. 
For nodes with deeper divergence times across Arthropoda, likelihood methods to reconstruct ancestral gene counts such as CAFE become inaccurate. Instead, a parsimony method was used to infer these gene counts across all 38,195 orthogroups [59]. Parsimony methods for gene family analysis do not include ways to assess significant changes in gene family size along a lineage. Hence, we performed a simple statistical test procedure for each branch to assess whether a given gene family was changing significantly: under a stochastic birth-death process of gene family evolution, and within a given family, the expected relationship between any node and its direct ancestor is that no change will have occurred. Therefore, we took all differences between nodes and their direct descendants in a family and compared them to a one-to-one linear regression. If any of the points differ from this oneto-one line by more than two standard deviations of the variance within the family, it was considered a significant change and that family is rapidly evolving along that lineage. Rates of gene gain and loss were estimated in a similar fashion to substitution rates. We counted the number of gene families inferred to be changing along each lineage and divided that by the estimated divergence time of that lineage (Fig. 4). To quantify the effect of any single species on the parsimony gene family reconstructions, we performed 100 jackknife replicates while randomly removing 5 species from each replicate. We find that ancestral gene counts are not greatly impacted by the presence or absence of any single genome (Additional file 2: Figure S14).

To estimate ancestral gene content (i.e., the number of genes at any given node in the tree), we had to correct for gene losses that are impossible to infer given the present data. To do this, we first regressed the number of genes at each internal node with the split time of that node and noticed the expected negative correlation of gene count and time (Additional file 2: Figure S1) $\left(r^{2}=\right.$ $\left.0.37 ; P=4.1 \times 10^{-9}\right)$. We then took the predicted value at time 0 (present day) as the number of expected genes if no unobserved gene loss occurs along any lineage and shifted the gene count of each node so that the residuals from the regression matched the residuals of the 0 value.

\section{Protein domain evolution analysis}

We annotated the proteomes of all 76 arthropod species and 13 outgroup species with protein domains from the Pfam database (v30) [60]. Thereby, every protein was represented as a domain arrangement, defined by its order of domains in the amino acid sequence. To prevent evaluating different isoforms of proteins as additional rearrangement events, we removed all but the longest isoform. Repeats of a same domain were collapsed to one instance of the domain (A-B-B-B-C $\rightarrow$ A$B-C)$, since copy numbers of some repeated domains can vary strongly even between closely related species [61, 62]. To be able to infer all rearrangement events over evolutionary time, we reconstructed the ancestral domain content of all inner nodes in the phylogenetic tree via the DomRates tool (http://domainworld.uni-muen ster.de/programs/domrates/) based on a combined parsimony approach (see Supplementary Methods). Six different event types were considered in this study (Additional file 2: Figure S15): fusion, fission, terminal loss/emergence, and single domain loss/emergence. For the rate calculation, just all arrangement changes were considered that could be explained by exactly one of these event types, while all arrangements were ignored that could not be explained by one of these events in a single step or if multiple events could explain a new arrangement.

\section{Supplementary information}

The online version of this article (https://doi.org/10.1186/s13059-019-1925-7) contains supplementary material, which is available to authorized users.

Additional file 1. Supplementary Tables S1 - S29.

Additional file 2. Supplementary Text and Supplementary Figures: Figures S1 - S34.

Additional file 3. Review history.

Acknowledgments

We thank the staff at the Baylor College of Medicine Human Genome Sequencing Center for their contributions.

Review history

The review history is available as Additional file 3.

\section{Authors' contributions}

GWCT performed phylogenetic analyses/reconstructions and designed the website. GWCT, ED, and RMW performed gene content and protein domain analysis and interpretation and contributed data to the website. KG and MADG performed methylation analysis. MB, HC, HD, HVD, SD, YH, JCJ, SLL, MM, NO-L, DMM, RAG, and SR managed and performed sequence library and sequence generation. SCM, JQ, DSTH, KCW, and SR performed genome assemblies, DSTH generated automated annotations, and DK, ES, and SR submitted data to public databases. MP, CC, and MM-T performed and supported manual annotation. NAA, JBB, DB, HC, JJD, LE, CEL, JG, RBG, CAA, PKK, NDY, PB, TP, DEG-R, AMH, MH, LH, WBH, AK, ARIL, GM, APM, DDM, BM, ON, SRP, KAP, MP, RSP, HCP, N-MP, DR, CS, SDS, EDS, DBS, RS, MRS, NUS, and EEZ provided species materials and expertise. RMW, PI, and EMZ performed orthology analysis. YL and MEP performed GO analysis, and AW, DDM, and MF assessed coleopteran and dipteran gene content change. JG summarized chelicerate gene families. MEP, KJH, JHW, KCW, GWCT, ED, RMW, ADC, EBB, $\mathrm{MWH}$, and SR prepared the manuscript contributed to project management and provided leadership. All authors read and approved the final manuscript.

\section{Funding}

Genome sequencing, assembly, and annotation were funded by National Human Genome Research Institute grant U54 HG003273 to R.A.G. GWCT and MWH are funded by NSF DBI-1564611. ED was funded by the Deutsche Forschungsgemeinschaft (DFG, German Research Foundation) - 281125614 / GRK2220. RMW, PI, and EMZ were funded by The Swiss National Science Foundation (PP00P3_170664 to RMW, 31003A_143936 to EMZ). Contributions by DDM and AW were supported in part by NSF-DEB grant 1355169 and USDA-APHIS Cooperative Agreement 15-8130-0547-CA to DDM. BM and ON acknowledge the German Research foundation (NI 1387/3-1, MI 649/12-1) and the Leibnitz Graduate School on Genomic Biodiversity Research. CS was supported by the Blanton J. Whitmire endowment, Housing and Urban 
Development NCHHU-0007-13, National Science Foundation 1557864 and Alfred P. Sloan Foundation 2013-5-35 MBE. Funding from Australian Wool Innovation (to P.B. and R.B.G.) and the Australian Research Council (to R.B.G.) is gratefully acknowledged. Support to R.B.G.'s laboratory by YourGene Bioscience and Melbourne Water Corporation is gratefully acknowledged. This project was also supported by a Victorian Life Sciences Computation Initiative (VLSCl; grant number VR0007) on its Peak Computing Facility at the University of Melbourne, an initiative of the Victorian Government (R.B.G.). C.A.A. holds an NSERC Postdoctoral Fellowship. N.D.Y. holds an NHMRC Early Career Research Fellowship. P.K.K. is the recipient of a scholarship (STRAPA) from the University of Melbourne. No funding body participated in the design of the study and collection, analysis, and interpretation of data and in writing the manuscript.

\section{Availability of data and materials}

All datasets generated and/or analyzed during the current study are publicly available. All reconstructed gene content for the lineages of the 76 species in this arthropod phylogeny are freely available at https://arthrofam.org and in Additional file 1: Table S11. All DNA, RNA, genome assembly, and transcriptome assembly sequences can be found at the NCBI, under the i5k Arthropod Genome Pilot Project (arthropods) Umbrella BioProject PRJNA163973 [63].

Full accessions for all data including SRA accessions for all sequence libraries, RNAseq libraries, assembly accessions for genome and transcriptomes are listed in Additional file 1: Tables S1-S4. Additional gene annotation data can be found at the USDA National Agricultural Libraries i5K workspace-for example the Asian longhorned beetle data is at https://i5k.nal.usda.gov/Anoplophora_glabripennis. Links for all automated annotations generated in this work are listed in Additional file 1: Table S5. Sources and versions for publicly available gene sets from previously sequenced species used in these analyses are listed in Additional file 1: Table S6.

\section{Ethics approval and consent to participate}

Not applicable.

\section{Consent for publication}

Not applicable.

\section{Competing interests}

The authors declare that they have no competing interests.

\section{Author details}

${ }^{1}$ Department of Biology and Department of Computer Science, Indiana University, Bloomington, IN, USA. ${ }^{2}$ Institute for Evolution and Biodiversity, University of Münsterss, 48149 Münster, Germany. ${ }^{3}$ Institute for Bioinformatics and Chemoinformatics, University of Hamburg, Hamburg, Germany. ${ }^{4}$ Westphalian University of Applied Sciences, 45665 Recklinghausen, Germany. ${ }^{5}$ Human Genome Sequencing Center, Department of Human and Molecular Genetics, Baylor College of Medicine, One Baylor Plaza, Houston, TX 77030, USA. ${ }^{6}$ Present Address: Institute for Genomic Medicine, Columbia University, New York, NY 10032, USA. ${ }^{7}$ Present Address: Howard Hughes Medical Institute, Department of Genome Sciences, University of Washington, Seattle, WA 98195, USA. ${ }^{8}$ National Agricultural Library, USDA, Beltsville, MD 20705, USA. ' School of Biological Sciences, Georgia Institute of Technology, Atlanta, GA 30332, USA. ${ }^{10}$ Present Address: Penn Epigenetics Institute, Department of Cell and Developmental Biology, University of Pennsylvania Perelman School of Medicine, Philadelphia, PA 19104, USA. ${ }^{11}$ Faculty of Veterinary and Agricultural Sciences, The University of Melbourne, Parkville, VIC 3010, Australia. ${ }^{12}$ Department of Biology, Washington and Lee University, 204 West Washington Street, Lexington, VA 24450, USA. ${ }^{13}$ School of BioSciences Science Faculty, The University of Melbourne, Melbourne, VIC 3010, Australia. ${ }^{14}$ Present Address: CooperGenomics, Houston, TX, USA. ${ }^{15}$ Department of Biology, Lewis \& Clark College, Portland, OR 97219, USA. ${ }^{16}$ Department of Plant and Soil Sciences, University of Vermont, Burlington, USA. ${ }^{17}$ Beneficial Insects Introduction Research Unit, United States Department of Agriculture, Agricultural Research Service, Newark, DE, USA. ${ }^{18}$ Institute for Biodiversity Science and Sustainability, California Academy of Sciences, 55 Music Concourse Drive, San Francisco, CA 94118, USA. ${ }^{19}$ Department of Biological Sciences, Wayne State University, Detroit, Ml 48202, USA. ${ }^{20}$ Department of Biological Sciences, University of Massachusetts Lowell, 198 Riverside Street, Lowell, MA 01854 ,
USA. ${ }^{21}$ USDA-ARS Invasive Insect Biocontrol and Behavior Laboratory, Beltsville, MD, USA. ${ }^{22}$ USDA-ARS, Center for Medical, Agricultural, and Veterinary Entomology, 1700 S.W. 23rd Drive, Gainesville, FL 32608, USA. ${ }^{23}$ Division of Insect Sciences, National Institute of Agrobiological Sciences, Owashi, Tsukuba 305-8634, Japan. ${ }^{24}$ Department of Zoology, Institute of Biology, University of Kassel, 34132 Kassel, Germany. ${ }^{25}$ USDA ARS, U. S. Horticultural Research Laboratory, Ft. Pierce, FL 34945, USA. ${ }^{26}$ Department of Genetic Medicine and Development and Swiss Institute of Bioinformatics, University of Geneva, 1211 Geneva, Switzerland. ${ }^{27}$ Present Address: Foundation for Research and Technology Hellas, Institute of Molecular Biology and Biotechnology, Vassilika Vouton, 70013 Heraklion, Greece. ${ }^{28}$ Université de Lyon, Institut de Génomique Fonctionnelle de Lyon, CNRS UMR 5242, Ecole Normale Supérieure de Lyon, Université Claude Bernard Lyon 1, 46 allée d'Italie, 69364 Lyon, France. ${ }^{29}$ Department of Integrative Biology, University of Wisconsin, Madison, WI 53706, USA. ${ }^{30}$ Department of Biological Sciences, University of Notre Dame, 109B Galvin Life Sciences, Notre Dame, IN 46556, USA. ${ }^{31}$ Department of Entomology, University of California Riverside, Riverside, CA, USA. ${ }^{32}$ Present Address: Department of Biology, Indiana University, Bloomington, IN, USA. ${ }^{33}$ Department of Biological and Medical Sciences, Oxford Brookes University, Gipsy Lane, Oxford OX3 OBP, UK. ${ }^{34}$ Department of Biological Sciences, University of Memphis, 3700 Walker Ave, Memphis, TN 38152, USA. ${ }^{35}$ Center for Molecular Biodiversity Research, Zoological Research Museum Alexander Koenig, Bonn, Germany. ${ }^{36}$ Environmental Genomics and Systems Biology Division, Lawrence Berkeley National Laboratory, Berkeley, USA. ${ }^{37}$ Present Address: Phoenix Bioinformatics, 39221 Paseo Padre Parkway, Ste. J., Fremont, CA 94538, USA. ${ }^{38}$ Evolutionary Biology and Ecology, Institute of Biology I (Zoology), Albert Ludwig University of Freiburg, 79104 Freiburg (Brsg.), Germany. ${ }^{39}$ Department of Entomology, University of Kentucky, Lexington, KY 40546, USA. ${ }^{40}$ School of Life Sciences, University of Warwick, Gibbet Hill Campus, Coventry CV4 7AL, UK. ${ }^{41}$ Cologne Biocenter, Zoological Institute, Department of Developmental Biology, University of Cologne, 50674 Cologne, Germany. ${ }^{42}$ Centre of Taxonomy and Evolutionary Research, Arthropoda Department, Zoological Research Museum Alexander Koenig, Bonn, Germany. ${ }^{43}$ School for the Environment, University of Massachusetts Boston, Boston, MA 02125, USA. ${ }^{44}$ Johann-Friedrich-Blumenbach-Institut für Zoologie und Anthropologie, Abteilung für Entwicklungsbiologie, Georg-August-Universität Göttingen, Göttingen, Germany. ${ }^{45}$ Göttingen Center for Molecular Biosciences (GZMB), Georg-August-Universität Göttingen, Göttingen, Germany. ${ }^{46}$ Department of Entomology and Plant Pathology, North Carolina State University, Raleigh, NC 27606, USA. ${ }^{47}$ Department of Entomology and W.M. Keck Center for Behavioral Biology, North Carolina State University, Raleigh, NC 27695, USA. ${ }^{48}$ Department of Entomology, University of Wisconsin-Madison, Madison, USA. ${ }^{49}$ Stored Product Insect and Engineering Research Unit, USDA-ARS Center for Grain and Animal Health Research, Manhattan, KS 66502, USA. ${ }^{50}$ Department of Biology, Colorado State University, Ft. Collins, CO, USA. ${ }^{51}$ Department of Entomology, University of Georgia, Athens, GA, USA. ${ }^{52}$ Present Address: Arkansas Biosciences Institute, Arkansas State University, Jonesboro, AR, USA. ${ }^{53}$ Natural History Museum Vienna, Burgring 7, 1010 Vienna, Austria. ${ }^{54}$ INIBIOMA, Univ. Nacional del Comahue - CONICET, Bariloche, Argentina. ${ }^{55}$ Department of Biological Sciences, University of Cincinnati, Cincinnati, $\mathrm{OH} 45221$, USA. ${ }^{56} \mathrm{Crop}$ Production and Protection, U.S. Department of Agriculture-Agricultural Research Service, Beltsville, MD 20705, USA. ${ }^{57}$ Department of Biology, University of Rochester, Rochester, NY 14627, USA. ${ }^{58}$ Department of Ecology, Evolution and Behavior, The Alexander Silberman Institute of Life Sciences, The Hebrew University of Jerusalem, Edmond J. Safra Campus, Givat Ram, 91904 Jerusalem, Israel. ${ }^{59}$ Department of Ecology \& Evolution and Swiss Institute of Bioinformatics, University of Lausanne, 1015 Lausanne, Switzerland. ${ }^{60}$ Department Protein Evolution, Max Planck Institute for Developmental Biology, Tübingen, Germany. ${ }^{61}$ Present Address: UC Davis Genome Center, University of California, Davis, CA 95616, USA.

Received: 6 November 2019 Accepted: 26 December 2019

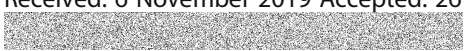

\section{References}

1. Lozano-Fernandez J, Carton R, Tanner AR, Puttick MN, Blaxter M, Vinther J, Olesen J, Giribet G, Edgecombe GD, Pisani D. A molecular palaeobiological exploration of arthropod terrestrialization. Philos Trans R Soc B. 2016;371: 20160133. 
2. Glenner H, Thomsen PF, Hebsgaard MB, Sorensen MV, Willerslev E. Evolution. The origin of insects. Science. 2006;314:1883-4.

3. Haug JT, Haug C, Garwood RJ. Evolution of insect wings and development new details from Palaeozoic nymphs. Biol Rev Camb Philos Soc. 2015;91:53-69.

4. Medved V, Marden JH, Fescemyer HW, Der JP, Liu J, Mahfooz N, Popadic A. Origin and diversification of wings: insights from a neopteran insect. Proc Natl Acad Sci U S A. 2015;112:15946-51.

5. Nel A, Roques P, Nel P, Prokin AA, Bourgoin T, Prokop J, Szwedo J, Azar D, Desutter-Grandcolas $L$, Wappler $T$, et al. The earliest known holometabolous insects. Nature. 2013;503:257-61.

6. i5K Consortium. The i5K Initiative: advancing arthropod genomics for knowledge, human health, agriculture, and the environment. J Hered. 2013; 104:595-600.

7. Zdobnov EM, Tegenfeldt F, Kuznetsov D, Waterhouse RM, Simao FA, loannidis P, Seppey M, Loetscher A, Kriventseva EV. OrthoDB v9.1: cataloging evolutionary and functional annotations for animal, fungal, plant, archaeal, bacterial and viral orthologs. Nucleic Acids Res. 2017:45:D744-9.

8. Misof B, Liu S, Meusemann K, Peters RS, Donath A, Mayer C, Frandsen PB, Ware J, Flouri T, Beutel RG, et al. Phylogenomics resolves the timing and pattern of insect evolution. Science. 2014;346:763-7.

9. Rota-Stabelli O, Daley AC, Pisani D. Molecular timetrees reveal a Cambrian colonization of land and a new scenario for ecdysozoan evolution. Curr Biol. 2013;23:392-8

10. Wipfler B, Letsch H, Frandsen PB, Kapli P, Mayer C, Bartel D, Buckley TR, Donath A, Edgerly-Rooks JS, Fujita M, et al. Evolutionary history of Polyneoptera and its implications for our understanding of early winged insects. Proc Natl Acad Sci U S A. 2019:116:3024-9.

11. Santos ME, Le Bouquin A, Crumiere AJJ, Khila A. Taxon-restricted genes at the origin of a novel trait allowing access to a new environment. Science. 2017:358:386-90.

12. Albalat R, Canestro C. Evolution by gene loss. Nat Rev Genet. 2016;17:379-91.

13. Porter ML, Crandall KA. Lost along the way: the significance of evolution in reverse. Trends Ecol Evol. 2003;18:541-7.

14. Lees JG, Dawson NL, Sillitoe I, Orengo CA. Functional innovation from changes in protein domains and their combinations. Curr Opin Struct Biol. 2016;38:44-52.

15. Eyun SI, Soh HY, Posavi M, Munro JB, Hughes DST, Murali SC, Qu J, Dugan S, Lee SL, Chao H, et al. Evolutionary history of chemosensory-related gene families across the Arthropoda. Mol Biol Evol. 2017;34:1838-62.

16. Arguello JR, Cardoso-Moreira M, Grenier JK, Gottipati S, Clark AG, Benton R. Extensive local adaptation within the chemosensory system following Drosophila melanogaster's global expansion. Nat Commun. 2016;7: ncomms11855.

17. Leal WS. Odorant reception in insects: roles of receptors, binding proteins, and degrading enzymes. Annu Rev Entomol. 2013;58:373-91.

18. Panfilio KA, Vargas Jentzsch IM, Benoit JB, Erezyilmaz D, Suzuki Y, Colella S, Robertson HM, Poelchau MF, Waterhouse RM, loannidis P, et al. Molecular evolutionary trends and feeding ecology diversification in the Hemiptera, anchored by the milkweed bug genome. Genome Biol. 2019;20:64.

19. Truman JW, Riddiford LM. The origins of insect metamorphosis. Nature. 1999:401:447-52

20. Harrison MC, Jongepier E, Robertson HM, Arning N, Bitard-Feildel T, Chao H, Childers CP, Dinh H, Doddapaneni H, Dugan S, et al. Hemimetabolous genomes reveal molecular basis of termite eusociality. Nat Ecol Evol. 2018;2:557-66.

21. Zhou X, Rokas A, Berger SL, Liebig J, Ray A, Zwiebel L. Chemoreceptor evolution in Hymenoptera and its implications for the evolution of Eusociality. Genome Biol Evol. 2015;7:2407-16.

22. Robertson HM, Baits RL, Walden KKO, Wada-Katsumata A, Schal C. Enormous expansion of the chemosensory gene repertoire in the omnivorous German cockroach Blattella germanica. J Exp Zool B Mol Dev Evol. 2018;300:265-78.

23. Wolfe JM, Daley AC, Legg DA, Edgecombe GD. Fossil calibrations for the arthropod tree of life. Earth Sci Rev. 2016;160:43-110.

24. Lee MS, Soubrier J, Edgecombe GD. Rates of phenotypic and genomic evolution during the Cambrian explosion. Curr Biol. 2013;23:1889-95.

25. Wissler L, Gadau J, Simola DF, Helmkampf M, Bornberg-Bauer E. Mechanisms and dynamics of orphan gene emergence in insect genomes. Genome Biol Evol. 2013;5:439-55.

26. Moore AD, Grath S, Schuler A, Huylmans AK, Bornberg-Bauer E. Quantification and functional analysis of modular protein evolution in a dense phylogenetic tree. Biochim Biophys Acta. 1834;2013:898-907.
27. Klasberg S, Bitard-Feildel T, Callebaut I, Bornberg-Bauer E. Origins and structural properties of novel and de novo protein domains during insect evolution. FEBS J. 2018;285:2605-25

28. Elango N, Hunt BG, Goodisman MA, Yi SV. DNA methylation is widespread and associated with differential gene expression in castes of the honeybee, Apis mellifera. Proc Natl Acad Sci U S A. 2009;106:11206-11.

29. International Aphid Genome Consortium. Genome sequence of the pea aphid Acyrthosiphon pisum. PLoS Biol. 2010;8:e1000313.

30. Provataris P, Meusemann K, Niehuis O, Grath S, Misof B. Signatures of DNA methylation across insects suggest reduced DNA methylation levels in Holometabola. Genome Biol Evol. 2018;10:1185-97.

31. Bewick AJ, Vogel KJ, Moore AJ, Schmitz RJ. Evolution of DNA methylation across insects. Mol Biol Evol. 2017;34:654-65.

32. Kent CF, Minaei S, Harpur BA, Zayed A. Recombination is associated with the evolution of genome structure and worker behavior in honey bees. Proc Natl Acad Sci U S A. 2012;109:18012-7.

33. Schoville SD, Chen $\mathrm{YH}$, Andersson MN, Benoit JB, Bhandari A, Bowsher JH, Brevik K, Cappelle K, Chen MM, Childers AK, et al. A model species for agricultural pest genomics: the genome of the Colorado potato beetle, Leptinotarsa decemlineata (Coleoptera: Chrysomelidae). Sci Rep. 2018:8:1931

34. Papanicolaou A, Schetelig MF, Arensburger P, Atkinson PW, Benoit JB, Bourtzis K, Castanera P, Cavanaugh JP, Chao H, Childers C, et al. The whole genome sequence of the Mediterranean fruit fly, Ceratitis capitata (Wiedemann), reveals insights into the biology and adaptive evolution of a highly invasive pest species. Genome Biol. 2016;17:192.

35. Anstead CA, Batterham P, Korhonen PK, Young ND, Hall RS, Bowles VM, Richards S, Scott MJ, Gasser RB. A blow to the fly - Lucilia cuprina draft genome and transcriptome to support advances in biology and biotechnology. Biotechnol Adv. 2016:34:605-20.

36. Benoit JB, Adelman ZN, Reinhardt K, Dolan A, Poelchau M, Jennings EC, Szuter EM, Hagan RW, Gujar H, Shukla JN, et al. Unique features of a global human ectoparasite identified through sequencing of the bed bug genome. Nat Commun. 2016;7:10165.

37. McKenna DD, Scully ED, Pauchet Y, Hoover K, Kirsch R, Geib SM, Mitchell RF, Waterhouse RM, Ahn SJ, Arsala D, et al. Genome of the Asian longhorned beetle (Anoplophora glabripennis), a globally significant invasive species, reveals key functional and evolutionary innovations at the beetle-plant interface. Genome Biol. 2016;17:227.

38. Lindsey ARI, Kelkar YD, Wu X, Sun D, Martinson EO, Yan Z, Rugman-Jones PF, Hughes DST, Murali SC, Qu J, et al. Comparative genomics of the miniature wasp and pest control agent Trichogramma pretiosum. BMC Biol. 2018;16:54

39. Armisen D, Rajakumar R, Friedrich M, Benoit JB, Robertson HM, Panfilio KA, Ahn SJ, Poelchau MF, Chao H, Dinh H, et al. The genome of the water strider Gerris buenoi reveals expansions of gene repertoires associated with adaptations to life on the water. BMC Genomics. 2018;19:832.

40. Schwager EE, Sharma PP, Clarke T, Leite DJ, Wierschin T, Pechmann M, Akiyama-Oda Y, Esposito L, Bechsgaard J, Bilde T, et al. The house spider genome reveals an ancient whole-genome duplication during arachnid evolution. BMC Biol. 2017;15:62

41. Poynton HC, Hasenbein S, Benoit JB, Sepulveda MS, Poelchau MF, Hughes DST, Murali SC, Chen S, Glastad KM, Goodisman MAD, et al. The Toxicogenome of Hyalella azteca: a model for sediment ecotoxicology and evolutionary toxicology. Environ Sci Technol. 2018;52:6009-22.

42. Petersen M, Armisen D, Gibbs RA, Hering L, Khila A, Mayer G, Richards S, Niehuis $\mathrm{O}$, Misof B. Diversity and evolution of the transposable element repertoire in arthropods with particular reference to insects. BMC Evol Biol. 2019:19:11.

43. Moore AD, Bornberg-Bauer E. The dynamics and evolutionary potential of domain loss and emergence. Mol Biol Evol. 2012;29:787-96.

44. Auman $\mathrm{T}$, Chipman AD. The evolution of gene regulatory networks that define arthropod body plans. Integr Comp Biol. 2017;57:523-32.

45. Davidson $\mathrm{EH}$. The regulatory genome: gene regulatory networks in development and evolution. San Diego, CA: Academic Press; 2006.

46. Maccallum I, Przybylski D, Gnerre S, Burton J, Shlyakhter I, Gnirke A, Malek J, McKernan K, Ranade S, Shea TP, et al. ALLPATHS 2: small genomes assembled accurately and with high continuity from short paired reads. Genome Biol. 2009:10:R103.

47. Butler J, MacCallum I, Kleber M, Shlyakhter IA, Belmonte MK, Lander ES, Nusbaum C, Jaffe DB. ALLPATHS: de novo assembly of whole-genome shotgun microreads. Genome Res. 2008;18:810-20. 
48. Atlas-Link [https://www.hgsc.bcm.edu/software/atlas-link]

49. ATLAS gapfill 2.2 [https://www.hgsc.bcm.edu/software/atlas-gapfill].

50. Pryszcz LP, Gabaldon T. Redundans: an assembly pipeline for highly heterozygous genomes. Nucleic Acids Res. 2016;44:e113.

51. Holt C, Yandell M. MAKER2: an annotation pipeline and genome-database management tool for second-generation genome projects. BMC Bioinformatics. 2011;12:491.

52. Poelchau M, Childers C, Moore G, Tsavatapalli V, Evans J, Lee CY, Lin H, Lin JW, Hackett K. The i5k workspace@NAL--enabling genomic data access, visualization and curation of arthropod genomes. Nucleic Acids Res. 2015:43:D714-9.

53. Waterhouse RM, Zdobnov EM, Tegenfeldt F, Li J, Kriventseva EV. OrthoDB: the hierarchical catalog of eukaryotic orthologs in 2011. Nucleic Acids Res. 2011;39:D283-8.

54. Kriventseva EV, Tegenfeldt F, Petty TJ, Waterhouse RM, Simao FA, Pozdnyakov IA, loannidis P, Zdobnov EM. OrthoDB v8: update of the hierarchical catalog of orthologs and the underlying free software. Nucleic Acids Res. 2015:43:D250-6.

55. Peters RS, Niehuis O, Gunkel S, Blaser M, Mayer C, Podsiadlowski L, Kozlov A, Donath A, van Noort S, Liu S, et al. Transcriptome sequence-based phylogeny of chalcidoid wasps (Hymenoptera: Chalcidoidea) reveals a history of rapid radiations, convergence, and evolutionary success. Mo Phylogenet Evol. 2018;120:286-96.

56. Schwentner M, Combosch DJ, Pakes Nelson J, Giribet G. A phylogenomic solution to the origin of insects by resolving crustacean-hexapod relationships. Curr Biol. 2017;27:1818-24. e1815

57. Sanderson MJ. r8s: inferring absolute rates of molecular evolution and divergence times in the absence of a molecular clock. Bioinformatics. 2003;19:301-2.

58. Han MV, Thomas GW, Lugo-Martinez J, Hahn MW. Estimating gene gain and loss rates in the presence of error in genome assembly and annotation using CAFE 3. Mol Biol Evol. 2013;30:1987-97.

59. Ames RM, Money D, Ghatge VP, Whelan S, Lovell SC. Determining the evolutionary history of gene families. Bioinformatics. 2012;28:48-55.

60. Finn RD, Coggill P, Eberhardt RY, Eddy SR, Mistry J, Mitchell AL, Potter SC, Punta M, Qureshi M, Sangrador-Vegas A, et al. The Pfam protein families database: towards a more sustainable future. Nucleic Acids Res. 2016;44:D279-85.

61. Schuler A, Bornberg-Bauer E. Evolution of protein domain repeats in Metazoa. Mol Biol Evol. 2016;33:3170-82.

62. Ekman D, Bjorklund AK, Elofsson A. Quantification of the elevated rate of domain rearrangements in metazoa. J Mol Biol. 2007;372:1337-48.

63. Thomas GWC, Dohmen E, Hughes DST, Murali SC, Poelchau M, Glastad K, et al. i5k arthropod genome pilot project. NCBI Seq Read Arch. https://www. ncbi.nlm.nih.gov/bioproject/?term=PRJNA163973

\section{Publisher's Note}

Springer Nature remains neutral with regard to jurisdictional claims in published maps and institutional affiliations.

\section{Ready to submit your research? Choose BMC and benefit from:}

- fast, convenient online submission

- thorough peer review by experienced researchers in your field

- rapid publication on acceptance

- support for research data, including large and complex data types

- gold Open Access which fosters wider collaboration and increased citations

- maximum visibility for your research: over $100 \mathrm{M}$ website views per year

At $\mathrm{BMC}$, research is always in progress.

Learn more biomedcentral.com/submissions 